\title{
Incremental Risk Charge Methodology
}

\author{
Tim Xiao ${ }^{1}$
}

\begin{abstract}
The incremental risk charge (IRC) is a new regulatory requirement from the Basel Committee in response to the recent financial crisis. Notably few models for IRC have been developed in the literature. This paper proposes a methodology consisting of two Monte Carlo simulations. The first Monte Carlo simulation simulates default, migration, and concentration in an integrated way. Combining with full re-valuation, the loss distribution at the first liquidity horizon for a subportfolio can be generated. The second Monte Carlo simulation is the random draws based on the constant level of risk assumption. It convolutes the copies of the single loss distribution to produce one year loss distribution. The aggregation of different subportfolios with different liquidity horizons is addressed. Moreover, the methodology for equity is also included, even though it is optional in IRC.
\end{abstract}

Keywords: Incremental risk charge (IRC), constant level of risk, liquidity horizon, constant loss distribution, Merton-type model, concentration.

\section{$1 \quad$ Introduction}

\footnotetext{
${ }^{1}$ Address correspondence to Tim Xiao, Risk Quant, Capital Markets, CIBC, 161 Bay Street, Toronto, ON M5J 2S8; email: tim_yxiao@yahoo.com Url: https://finpricing.com/
} 
The Basel Committee on Banking Supervision (see Basel [2009 a]) released the new guidelines for Incremental Risk Charge (IRC) that are part of the new rules developed in response to the financial crisis and is a key part of a series of regulatory enhancements being rolled out by regulators.

IRC supplements existing Value-at-Risk (VaR) and captures the loss due to default and migration events at a $99.9 \%$ confidence level over a one-year capital horizon. The liquidity of position is explicitly modeled in IRC through liquidity horizon and constant level of risk.

The constant level of risk assumption in IRC reflects the view that securities and derivatives held in the trading book are generally more liquid than those in the banking book and may be rebalanced more frequently than once a year. IRC should assume a constant level of risk over a one-year capital horizon which may contain shorter liquidity horizons. This constant level of risk assumption implies that a bank would rebalance, or rollover, its positions over the oneyear capital horizon in a manner that maintains the initial risk level, as indicated by the profile of exposure by credit rating and concentration.

The current market risk capital rule is:

\section{Total market risk capital $=$ general market risk capital \\ + basic specific risk capital \\ + specific risk surcharge}

where

General market risk capital $=3 \times$ General_ $V_{-} R_{99 \%}^{10-d a y}$

Basic specific risk capital $=3 \times$ Specific_VaR $R_{99 \%}^{10-d a y}$

Specific risk surcharge $=(\mathrm{m}-3) \times$ Specific_VaR $R_{99 \%}^{10-d a y}$

where $m$ is the specific risk capital multiplier under regulators' guidance

The new market risk capital standard will be: 


\section{Total market risk capital $=$ general market risk capital \\ + basic specific risk capital \\ + incremental risk charge}

where Incremental risk charge $=I R C_{-} V a R_{99.9 \%}^{1-y e a r}$

In this paper, we present a methodology for calculating IRC. First, a Merton-type model is introduced for simulating default and migration. The model is modified to incorporate concentration. The calibration is also elaborated. Second, a simple approach to determine market data, including equity, in response to default and credit migration is presented. Next, a methodology toward constant level of risk is described. The details of applying the constant level of risk assumption and aggregating different subportfolios are addressed. Finally, the empirical and numerical results are presented.

\section{Simulation of Default and Credit Migration}

The IRC encompasses all positions subject to a capital charge for specific interest rate risk according to the internal models with exception of securitization and nth-to-default credit derivatives. Equity is optional. For IRC-covered positions, the IRC captures default risk and credit migration risk only.

\subsection{Simulation Model}

Most of the portfolio models of credit risk used in the banking industry is based on the conditional independence framework. In these models, defaults and credit migration of individual borrowers depend on a set of common systematic risk factors describing the state of the economy. Merton-type models have become very popular. The Merton-type model (or standardized Merton model) is

$$
z_{i}=\beta_{i} \phi+\sqrt{1-\beta_{i}^{2}} \varepsilon_{i}
$$

where 
$\phi, \varepsilon_{i} \quad$ The independent standard normally random variables

$\phi \quad$ The systematic risk

$\varepsilon_{i} \quad$ The idiosyncratic risk for issuer/obligor i

$\beta_{i} \quad$ The weighted correlation reflecting the impact of systematic risk factor on issuer/obligor $\mathrm{i}$.

$z_{i} \quad$ The normalized asset return or creditworthiness indicator for issuer/obligor i

This model becomes the most popular one in default and migration risk modeling and yields the core of the Basel II capital rule (see Heitfield [2003]).

Similar to the original Merton model, this model is also assuming that the default and migration only happens at the end, which achieves significant simplification.

\subsection{Simulation model for multiple-liquidity-horizon subportfolios}

Liquidity horizons are determined for each position to reflect actual practice and experience during periods of both systematic and idiosyncratic stresses. The total portfolio shall be divided into the subportfolios based on different liquidity horizons. Let's assume that there are two subportfolios with different liquidity horizons: 3 month and 6 month. To model different liquidity periods, one can use the above model (3) but calibrate different $\beta_{i}$ 's, such as, $\beta_{3 m_{-} i}$ and $\beta_{6 m_{-} i}$, for different periods.

Alternatively, one can also use a multiple-period model as:

$$
\begin{array}{ll}
z_{3 m}=\beta_{i} \phi_{3 m}+\sqrt{1-\beta_{i}^{2}} \varepsilon_{3 m_{-} i} & \text { For 3 month } \\
z_{6 m}=\beta_{i} \frac{\phi_{6 m}+\gamma \cdot \phi_{3 m}}{\sqrt{1+\gamma^{2}}}+\sqrt{1-\beta_{i}^{2}} \varepsilon_{6 m_{-} i} & \text { For 6 month }
\end{array}
$$

where $\beta_{i}$ is unique for different periods under issuer $\mathrm{i}$ and $\gamma$ is an exponentially declining weight (see Dunn [2008]). 


\subsection{Calibration of $\beta_{i}$}

The most popular approaches to calibrate the asset correlation are Maximum Likelihood Estimation or regression based on time series default data. Alternatively, in the new Basel Capital Accord, a formula for derivation of risk weighted asset correlation for corporate, sovereign, and bank exposures is given as (see Tasche [2004] and Basel [2003]):

$$
\beta_{i}=0.12 \times \lambda_{i}+0.24 \times\left(1-\lambda_{i}\right)
$$

Where $\lambda_{i}=\frac{1-e^{-50 \times P D_{i}}}{1-e^{-50}}$

\subsection{Concentration}

The phenomenon we need to model is that concentration will result a higher IRC number, comparing to non-concentration case. Furthermore, the more concentration a portfolio has, the higher IRC result it generates. To achieve this, we model the effect of issuer and market concentration as well as clustering of default and migration by introducing another parameter, the

concentration parameter.

There are two correlations we need to consider: correlation between credit migration and default events of obligors and correlation between credit migration/default events and systematic market risk factors. The study (see Kim [2009]) shows that the correlation between credit migration/default events and systematic market risk factors is very small and negligible. However, correlation between credit migration and default events of obligors is significant and cannot be ignored. Therefore, the concentration parameter is solely dependent on correlation between credit migration and default.

Our methodology is based on a simple mechanism for coupling issuer/market concentrations to migrations and defaults. In the simulation framework (3) or (4) and (5), the probability of a migration or default increases with the asset volatility. Since the effect of increasing concentration within a sector is to increase the probability of migration/default events 
within that sector, we model increased concentration as an increase in the volatility of the systematic risk driver. All positions sensitive to that risk driver will have an increased probability of migration/default events occurring. The modified simulation model is

$$
z_{i}=\beta_{i}\left(1+\sqrt{\left|\rho_{i}\right|}\right) \phi_{t}+\sqrt{1-\beta_{i}^{2}} \varepsilon_{i}
$$

Where $\rho_{i}$ is the weighted concentration factor depending on correlation between issuer default and migration events and

$$
\phi_{t}=\frac{x_{t}+\gamma \cdot x_{t-1}+\cdots+\gamma^{k} \cdot x_{t-k}}{\sqrt{1+\gamma^{2}+\cdots+\gamma^{2 k}}}
$$

where if one uses (3), $\gamma=0$ and $\phi_{t}=x_{t}=\phi$. Otherwise, $\gamma$ is time declining weight and $x_{t}, \cdots, x_{t-k}$ are independent standard normally random variables representing systematic risks in different time periods.

\section{$2.5 \quad$ Calibration of $\rho_{i}$}

The calibration is based on credit migration matrix. It can be derived using either analytic closed-form or Monte-Carlo simulation. In theory, one can use Pearson's product moment or Kendall's $\tau$.

\subsection{Determination of default and credit migration}

The simulated asset return $z_{i}$, combined with migration/default thresholds, is used to ascertain when default or migration is deemed to occur. The calculation of the thresholds of credit migration and default is based on credit migration probability (see JP Morgan [1997]). Using a $\mathrm{BBB}$ issuer as an example and given migration matrix, we can calculate the thresholds as: $z_{D}^{B B B}, z_{C C C}^{B B B}, z_{B}^{B B B}, z_{B B}^{B B B}, z_{B B B}^{B B B}, z_{A}^{B B B}, z_{A A}^{B B B}$. The rating bands and thresholds are shown in Figure 1 


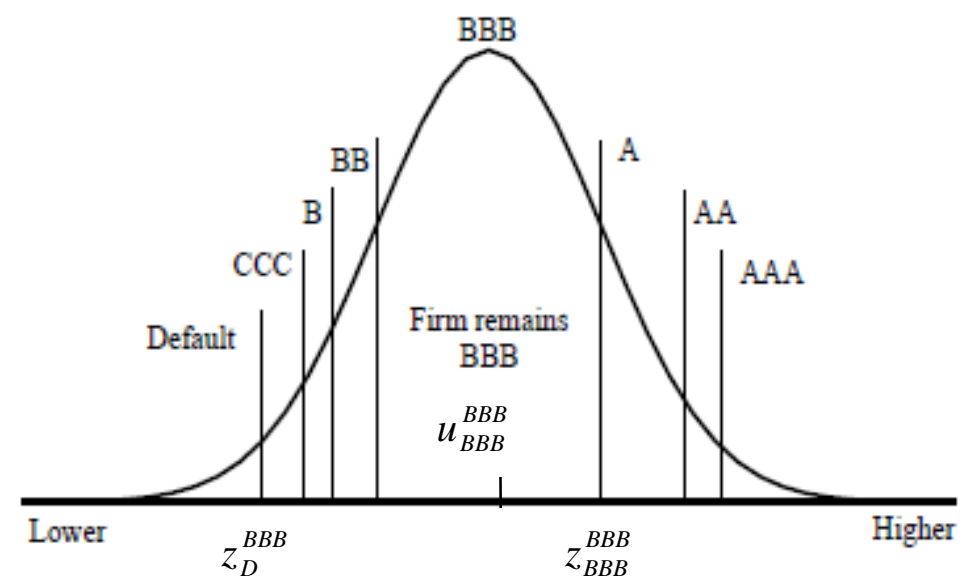

Figure 1 Credit migration rating thresholds (for BBB)

If the normalized asset of the issuer is smaller than $z_{D}^{B B B}$, it defaults. If the normalized asset is between $z_{D}^{B B B}$ and $z_{C C C}^{B B B}$, it migrates to CCC, and so on. We use an effective middle value to represent each band:

$$
\begin{aligned}
& u_{D}^{B B B}=\Phi^{-1}\left[\frac{1}{2}\left(\left(\Phi\left(z_{D}^{B B B}\right)+0\right)\right]\right. \\
& u_{C C C}^{B B B}=\Phi^{-1}\left[\frac{1}{2}\left(\left(\Phi\left(z_{C C C}^{B B B}\right)+\Phi\left(z_{D}^{B B B}\right)\right)\right]\right. \\
& u_{B}^{B B B}=\Phi^{-1}\left[\frac{1}{2}\left(\left(\Phi\left(z_{C C C}^{B B B}\right)+\Phi\left(z_{B}^{B B B}\right)\right)\right]\right. \\
& u_{B B}^{B B B}=\Phi^{-1}\left[\frac{1}{2}\left(\left(\Phi\left(z_{B}^{B B B}\right)+\Phi\left(z_{B B}^{B B B}\right)\right)\right]\right. \\
& u_{B B B}^{B B B}=\Phi^{-1}\left[\frac{1}{2}\left(\left(\Phi\left(z_{B B}^{B B B}\right)+\Phi\left(z_{B B B}^{B B B}\right)\right)\right]\right. \\
& u_{A}^{B B B}=\Phi^{-1}\left[\frac{1}{2}\left(\left(\Phi\left(z_{B B B}^{B B B}\right)+\Phi\left(z_{A}^{B B B}\right)\right)\right]\right. \\
& u_{A A}^{B B B}=\Phi^{-1}\left[\frac{1}{2}\left(\left(\Phi\left(z_{A}^{B B B}\right)+\Phi\left(z_{A A}^{B B B}\right)\right)\right]\right. \\
& u_{A A A}^{B B B}=\Phi^{-1}\left[\frac{1}{2}\left(\left(\Phi\left(z_{A A}^{B B B}\right)+1\right)\right]\right.
\end{aligned}
$$

2.7 Calibration of transition matrix, default probability (PD), and loss given default (LGD) 
The straight forward cohort approach is used to estimate transition matrices based on obligors' rating history, which has become the industry standard. The PD estimate is based on EDF data that is used for calculation of PD benchmarked against internal default history. Internal data is used for LGD parameter benchmarked against relevant external proxy data.

\section{Credit Spreads and Equity Prices}

After simulating default and migration of all issuers/obligors, we need to price every instrument in order to generate loss distributions. The question is whether we should simulate market data or not?

The earlier version of Basel IRC paper (see Basel [2008]) requires financial institutes to capture four risks: default, credit migration, significant credit spread changes, and significant equity price changes. However, the new guideline (see Basel [2009 a]) limits the risks to default and credit migration only. In addition, a separate Basel paper (see Basel [2009 b]) further states that IRC contains only incremental default and migration risks, and all price risks belong to the comprehensive risk. These messages give us a clear indication that only default and credit migration are risk factors in IRC and all market prices/data are not. Therefore, we recommend simulating default and migration only but not simulating any market prices/data.

We assume all market prices/data are deterministic (flat) and use forward prices/data for valuation. The fat tail behavior and market correlations are embedded in the market. Keeping these parameters constant ensures we measure only $P \& L$ variation due to credit rating changes (migration or default) per IRC requirements. The selection of credit spreads or equity prices, however, should reflect the credit quality changes.

\subsection{Credit spreads}


All issuers/obligors shall be divided into credit groups based on geographies and sectors. Assume that the credit spreads for different ratings under each group are available. Then we can select associated credit spreads to price a bond or a CDS according to the creditworthiness simulation of the issuer/obligor.

\subsection{Equity prices}

In risk neutral world, the forward equity price at future time $\mathrm{T}$ is

$$
E_{T}=E_{0} e^{r T}
$$

Where $r$ is the risk free interest rate and $E_{0}$ is the today's spot equity price

If the issuer defaults at $T$, the equity price should be 0 . If the issuer is upgraded or downgraded, the equity price should be larger or smaller than the risk neutral forward price $E_{T}=E_{0} e^{r T}$. This is the phenomenon we are going to model:

$$
E_{T}=\left\{\begin{array}{lr}
E_{0} e^{r T} & \text { if no credit change } \\
0 & \text { if default } \\
>E_{0} e^{r T} & \text { if upgraded } \\
<E_{o} e^{r T} & \text { if downgraded }
\end{array}\right.
$$

The underlying dynamic of Merton model is

$$
d A_{t}=r A_{t} d t+\sigma_{A} A_{t} d W_{t}
$$

Where $A_{t}$ is the corporate asset value; $r$ is the risk-free interest rate; $\sigma_{A}$ is the asset volatility

and $W_{t}$ is the Wiener process.

Applying Ito's lemma, we have

$$
A_{T}=A_{0} \exp \left(r T-\frac{1}{2} \sigma_{A}^{2} T+\sigma_{A} \sqrt{T} y\right)
$$

where y denote the standard normal variable

The Merton model states that the equity of a company is a European call option on the asset of the company with maturity $\mathrm{T}$ and a strike price equal to the face value of the debt that will become due at $T$. 
The payoff of Merton model is

$$
E_{T}=\max \left(A_{T}-D, 0\right)
$$

where $D$ denotes the debt of the company.

The mathematical expression of Merton model is

$$
E_{0}=A_{0} N\left(d_{1}\right)-e^{-r T} D N\left(d_{2}\right)
$$

where $d_{1,2}=\frac{\ln \left(A_{0} / D\right)+r T}{\sigma_{A} \sqrt{\tau}} \pm \frac{1}{2} \sigma_{A} \sqrt{T}$

We still use the BBB issuer as an example. Based on (8), (12), and (13), the equity price at $\mathrm{T}$, if default occurs, is

$$
E_{T}^{B B B->D}=A_{T}^{D}-D=A_{0} \exp \left(r T-\frac{1}{2} \sigma_{A}^{2} T+\sigma_{A} \sqrt{T} u_{D}^{B B B}\right)-D=0
$$

The equity price at $\mathrm{T}$ without credit quality changes is

$$
E_{T}^{B B B->B B B}=A_{T}^{B B B}-D=A_{0} \exp \left(r T-\frac{1}{2} \sigma_{A}^{2} T+\sigma_{A} \sqrt{T} u_{B B B}^{B B B}\right)-D=E_{0} e^{r T}
$$

We solve equations (14), (15), and (16) to get $A_{0}, \sigma_{A}$, and D. Then, with the known $A_{0}$, $\sigma_{A}$, and $\mathrm{D}$, we can obtain any equity price at $\mathrm{T}$ under any credit rating according to (8) and (13). For instance, when the rating changes from $\mathrm{BBB}$ to $\mathrm{A}$, the equity price at $\mathrm{T}$ is

$$
E_{T}^{B B B->A}=\max \left(A_{T}^{A}-D, 0\right)=A_{0} \exp \left(r T-\frac{1}{2} \sigma_{A}^{2} T+\sigma_{A} \sqrt{T} u_{A}^{B B B}\right)-D
$$

\section{Constant Level of Risk}

The constant level of risk reflects recognition by regulators that securities/derivatives held in the trading book are generally much more liquid than those in the banking book, where a buy-and-hold assumption over one year may be reasonable. It implies that IRC should be modeled under the assumption that banks rebalance their portfolio several times over the capital horizon in order to maintain a constant risk profile as market conditions evolve. Of course, we do not suggest that the constant level of risk framework be taken literally as a model of banks' 
behavior: clearly portfolios are altered on a daily basis, not simply held constant for some period then instantaneously rebalanced. Rather, we regard the rollover interpretation as being a reasonable approximation to the way banks manage their trading portfolios over a certain horizon. In general, one should model constant level of risk instead of constant portfolio over one year capital horizon.

There are several ways to interpret constant level of risk: constant loss distribution or constant risk metrics (e.g. VaR). We believe the constant loss distribution assumption is the most rigorous. Under this assumption, the same metrics (e.g. VaR, moments, etc.) can be achieved for each liquidity horizon.

The liquidity horizon for a position or set of positions has a floor of three months. Let us use three months as an example. We interpret constant level of risk to mean that the bank holds its portfolio constant for the liquidity horizon, then rebalances by selling any default, downgraded, or upgraded positions and replaces them so that the portfolio is returned to the level of risk it had at the beginning. The process is repeated 4 times over the capital horizon resulting 4 independent and identical loss distributions. The one year constant level of risk loss distribution is the convolution of 4 copies of the three month loss distribution. In Monte Carlo context, this can be modeled by drawing 4 times from the single period loss distribution measured over the liquidity horizon. The total PnL is the summary of these 4 random draws.

An intuitive explanation is shown in Figure 2. A generic path with appears in red; P\&L contributions from each liquidity horizon appear in blue. In this schematic, the position experiences downgrade, upgrade or default, resulting in a loss or profit. This position is then removed and replaced at the end of each liquidity horizon by rebalancing. The final P\&L for the path will be the summary of all losses and profits.

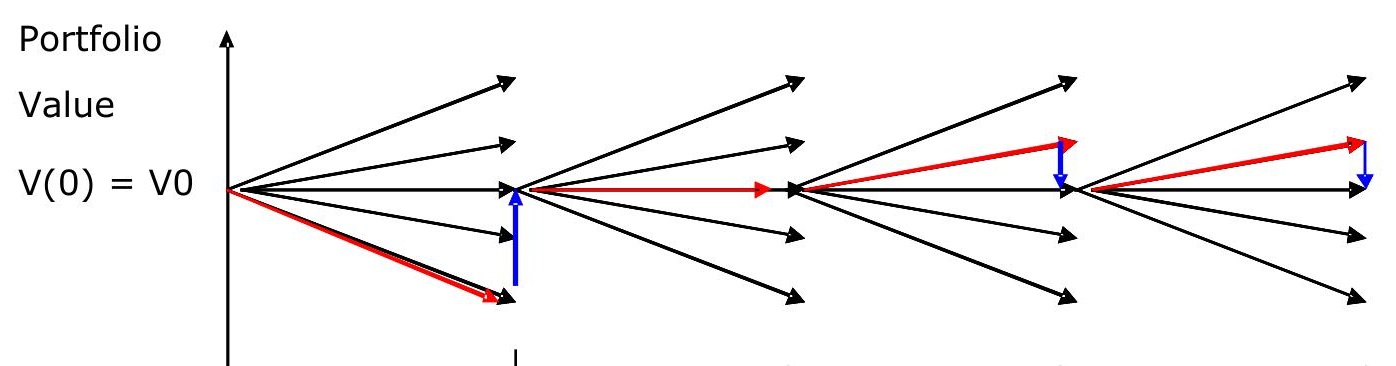


Figure 2 Constant level of risk

In addition, one needs to consider the reinvestment of all cash flows realized during the liquidity horizon and rollover of expired deals.

\section{$5 \quad$ Aggregation and Time Horizon Correlation}

First we need to divide the portfolio into the subportfolios based on liquidity horizons. If there is only one single-liquidity-horizon subportfolio, the rebalance at the end of each liquidity horizon washes out the time horizon correlation. However, if there are multiple subportfolios, the time horizon correlations need to be addressed.

To elaborate the details, we assume there are two subportfolios with liquidity horizons: 3 months and 6 months. Based on the default and migration simulation and full re-valuation, we can generate loss distributions at first liquidity horizons for 3-month and 6-month subportfolios as $P L_{3 m}$, and $P L_{6 m}$.

There are two approaches to achieve the correlative aggregation: copula approach or correlation matrix approach.

\section{$5.1 \quad$ Copula approach}


We conduct the second Monte Carlo simulation by generate 4 standard normal random draws for scenario $\mathrm{j}: x_{1}^{j}, x_{2}^{j}, . x_{3}^{j}, x_{4}^{j}$. These random draws represent a Monte-Carlo path.

\subsubsection{Three-month Subportfolio}

The P\&L distribution of three-month subportfolio is $P L_{3 m}$. The four draws of loss distribution are $P L_{3 m}\left(\Phi\left(x_{1}^{j}\right)\right), P L_{3 m}\left(\Phi\left(x_{2}^{j}\right)\right), P L_{3 m}\left(\Phi\left(x_{3}^{j}\right)\right), P L_{3 m}\left(\Phi\left(x_{4}^{j}\right)\right)$, where $\Phi$ is the accumulative normal. The total $P \& L$ of the three-month subportfolio for scenario $j$ is

$$
P L_{\text {total_3m }}^{j}=\sum_{i=1}^{4} P L_{3 m}\left(\Phi\left(x_{i}^{j}\right)\right)
$$

\subsubsection{Six-month Subportfolio}

The P\&L distribution of the six-month subportfolio is $P L_{6 m}$. We can calculate correlation $\rho\left(P L_{3 m}, P L_{6 m}\right)$ between $P L_{3 m}$ and $P L_{6 m}$ using Pearson product-moment. The two correlated random draws are $x_{6 m_{-} 1}^{j}=\rho\left(P L_{3 m}, P L_{6 m}\right) x_{1}^{j}+\sqrt{1-\rho\left(P L_{3 m}, P L_{6 m}\right)^{2}} x_{2}^{j} \quad$ and $x_{6 m_{-} 2}^{j}=\rho\left(P L_{3 m}, P L_{6 m}\right) x_{3}^{j}+\sqrt{1-\rho\left(P L_{3 m}, P L_{6 m}\right)^{2}} x_{4}^{j}$. The two draws of loss distribution are $P L_{6 m}\left(\Phi\left(x_{6 m_{-} 1}^{j}\right)\right), P L_{6 m}\left(\Phi\left(x_{6 m_{-} 2}^{j}\right)\right)$. The total P\&L of the six-month subportfolio for scenario $\mathrm{j}$ is

$$
P L_{\text {total } \_6 m}^{j}=\sum_{i=1}^{2} P L_{6 m}\left(\Phi\left(x_{6 m_{-} i}^{j}\right)\right)
$$

Summing up (18) and (19), we can get the total P\&L for scenario $\mathrm{j}$ as

$$
P L_{\text {total }}^{j}=P L_{\text {total_ } 6 m}^{j}+P L_{\text {total } \_3 m}^{j}
$$

\subsection{Correlation matrix approach}

Based on the four 3-month independent identical loss distributions: $P L_{3 m}, P L_{3 m}, P L_{3 m}, P L_{3 m}$, and two 6-month independent identical loss distributions: $P L_{6 m}, P L_{6 m}$, we can construct a $6 \times 6$ pair-wise sample correlation matrix $\Sigma$. Applying the 
Cholesky decomposition to the correlation matrix $\Sigma$, we have $\Sigma=L L^{T}$, where $L$ is a lower triangular matrix.

We conduct the second Monte Carlo simulation by generating 4 independent standard normal random draws: $x_{1}^{j}, x_{2}^{j}, . x_{3}^{j}, x_{4}^{j}$ for the four 3-month periods in a year and 2 independent standard normal random draws $x_{5}^{j}, x_{6}^{j}$ for the two 6-month periods to construct a path/scenario $\mathrm{j}$. The random draw vector is $X=\left[\begin{array}{llllll}x_{1}^{j} & x_{2}^{j} & x_{3}^{j} & x_{4}^{j} & x_{5}^{j} & x_{6}^{j}\end{array}\right]$. We can obtain correlative random draw vector

$$
\tilde{X}=\left[\begin{array}{llllll}
\tilde{x}_{1}^{j} & \tilde{x}_{2}^{j} & \tilde{x}_{3}^{j} & \tilde{x}_{4}^{j} & \tilde{x}_{5}^{j} & \tilde{x}_{6}^{j}
\end{array}\right] \text { by } \tilde{X}^{T}=L \times X^{T}
$$

The total P\&L for scenario $\mathrm{j}$ is

$$
P L_{\text {total }}^{j}=P L_{\text {total_3m }}^{j}+P L_{\text {total } \_6 m}^{j}=\sum_{i=1}^{4} P L_{3 m}\left(\Phi\left(\tilde{x}_{i}^{j}\right)\right)+\sum_{i=5}^{6} P L_{6 m}\left(\Phi\left(\tilde{x}_{i}^{j}\right)\right)
$$

The final IRC will be $99.9 \% \mathrm{VaR}$ based on distribution $P L_{\text {total }}^{j}$. In general, the correlation matrix approach is more generic and can be easily extended to any number of subportfolios.

\section{$6 \quad$ Numerical and Empirical Results}

The above methodology has been implemented. The empirical study shows the results on P\&L distributions, numerical stability \& convergence, concentration effect, and capital impact.

The loss distributions for the testing portfolio are shown in Figure 3 and 4. 


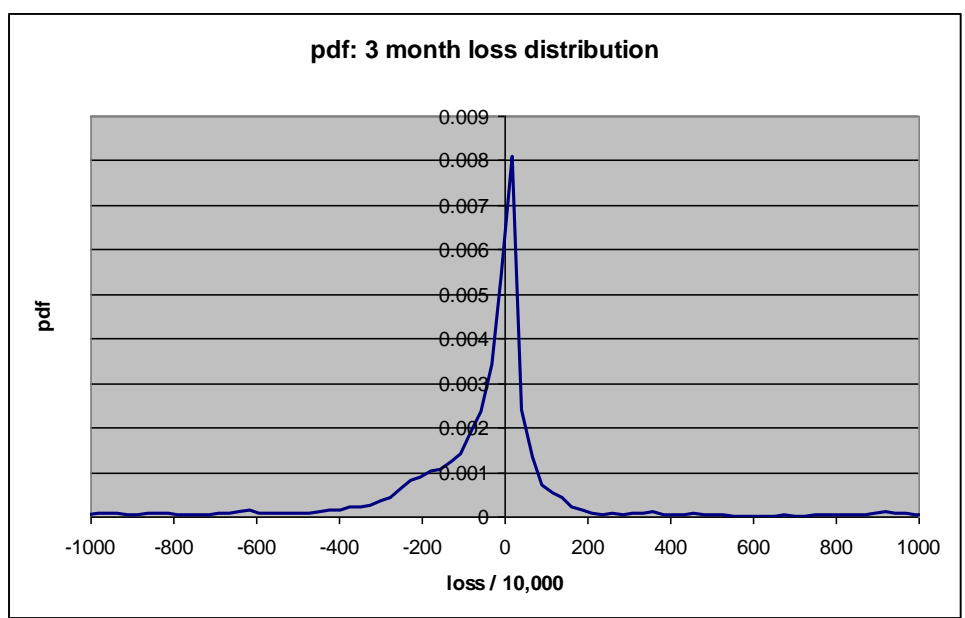

Figure 3 Histogram of loss distribution at 3 month

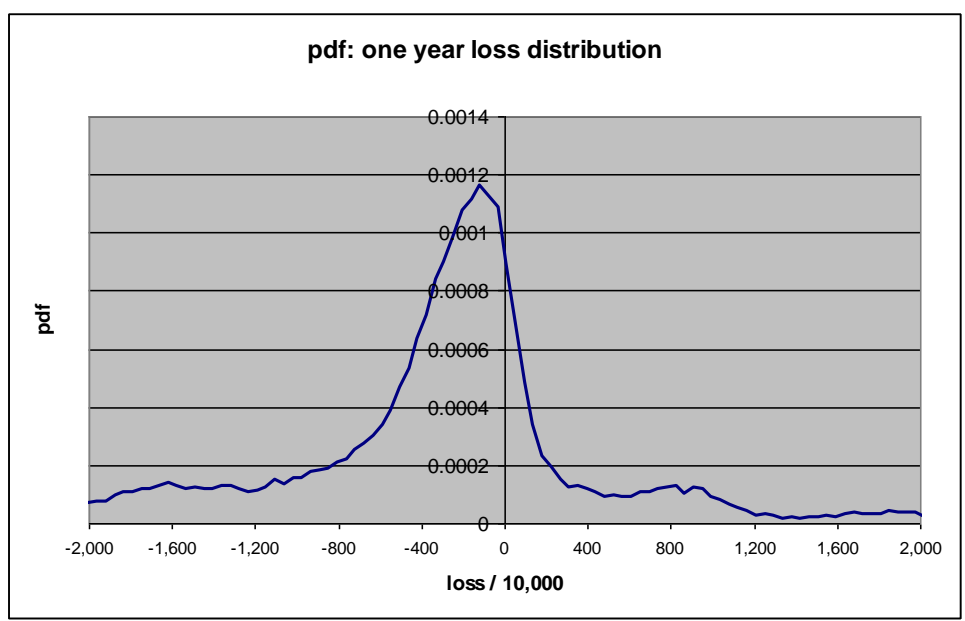

Figure $4 \quad$ Histogram of loss distribution at 1 year

\subsection{Convergence study}

People normally believe that 50,000 simulations provide sufficient stability to measure the $99.9^{\text {th }}$ percentile loss required for the regulatory IRC measure. However, our study shows that 50,000 paths are not convergent. Actually 100,000 simulations are needed to archive a better numerical stability and convergence. The results are shown in Table 1

Table 1 convergence results 


\begin{tabular}{|c|c|c|c|}
\hline Scenarios & IRC & Diff from previous & Diff from average \\
\hline $8,000.00$ & 102.31 & & $-1.51 \%$ \\
\hline $10,000.00$ & 103.56 & $1.23 \%$ & $-0.30 \%$ \\
\hline $20,000.00$ & 100.44 & $-3.01 \%$ & $-3.30 \%$ \\
\hline $40,000.00$ & 100.71 & $0.27 \%$ & $-3.04 \%$ \\
\hline $60,000.00$ & 110.01 & $9.23 \%$ & $5.91 \%$ \\
\hline $80,000.00$ & 105.22 & $-4.35 \%$ & $1.30 \%$ \\
\hline $100,000.00$ & 104.90 & $-0.31 \%$ & $0.99 \%$ \\
\hline $120,000.00$ & 103.66 & $-1.18 \%$ & $-0.20 \%$ \\
\hline $140,000.00$ & 103.96 & $0.28 \%$ & $0.08 \%$ \\
\hline $160,000.00$ & 103.61 & $-0.33 \%$ & $-0.25 \%$ \\
\hline $180,000.00$ & 105.23 & $1.56 \%$ & $1.31 \%$ \\
\hline $200,000.00$ & 103.14 & $-1.99 \%$ & $-0.71 \%$ \\
\hline Average & 103.87 & & \\
\hline
\end{tabular}

\subsection{Concentration study}

The purpose of this section is to demonstrate that the model (7) can reflect issuer and market concentrations. To simplify our tests, we assign all issuers with the same concentration factor $\rho$. It is shown that the IRC increases according to the increasing of $\rho$, up to $30 \%$ in table 2.

Table 2 Concentration study

\begin{tabular}{|r|r|r|rr|}
\hline Scenarios & $\rho$ & & IRC & Diff from 0 concentration \\
\hline 100,000 & 0 & 104.90 & 0 \\
\hline 100,000 & 0.2 & 116.97 & $11.50 \%$ \\
\hline
\end{tabular}




\begin{tabular}{|r|r|r|r|}
\hline 100,000 & 0.4 & 122.37 & $16.66 \%$ \\
\hline 100,000 & 0.6 & 128.49 & $22.48 \%$ \\
\hline 100,000 & 0.8 & 132.83 & $26.63 \%$ \\
\hline 100,000 & 1 & 137.23 & $30.82 \%$ \\
\hline
\end{tabular}

\subsection{Capital impact}

The capital impact can be measured as the ratio between IRC and specific risk surcharge. The results significantly depend on the composition of a portfolio and the specific risk multiplier of a financial institution set by the regulator. The ratio of our testing portfolio is 5.8 .

\section{References}

Alessandro Aimone, 2018, “ING's market risk charge edges higher,” Risk Quantum, 2018

Basel Committee on Banking Supervision, 31 July 2003, "The new Basel capital accord.”

Basel Committee on Banking Supervision, July 2008, "Guidelines for Computing Capital for Incremental Default Risk in the Trading Book.”

Basel Committee on Banking Supervision, July 2009 (a), "Guidelines for Computing Capital for Incremental Risk in the Trading Book."

Basel Committee on Banking Supervision, July, 2009 (b), "Revisions to the Basel II market risk framework." 
Basel Committee on Banking Supervision, October 2009 C, "Analysis of the trading book quantitative impact study."

Gary Dunn, April 2008, “A multiple period Gaussian Jump to Default Risk Model.”

FinPricing, Risk Management Solution, https://finpricing.com/paperList.html

Erik Heitfield, 2003, "Dealing with double default under Basel II," Board of Governors of the Federal Reserve System.

Jongwoo Kim, Feb 2009, "Hypothesis Test of Default Correlation and Application to Specific Risk,” RiskMetrics Group.

J.P.Morgan, April, 1997, “CreditMetrics - Technical Document.”

Dirk Tasche, Feb 17, 2004, "The single risk factor approach to capital charges in case of correlated loss given default rates.”

Tim Xiao, February 2009, “Incremental Risk Charge Methodology,” CIBC Internal.

Tim Xiao, etc, May 2009, “Incremental Risk Charge Impact Study,” CIBC Internal. 